\section{俩 Heighten Science P U B L I C I T I O N S Corporation ISSN 2637-3793}

\title{
A rare case: Congenital Megalourethra in prune belly syndrome
}

\author{
Ismail Selvi ${ }^{1}$, Numan Baydilli2* and Emre Can Akınsal ${ }^{2}$ \\ 'Department of Urology, Dr. Abdurrahman Yurtaslan Ankara Oncology Training and Research \\ Hospital, Ankara, Turkey \\ ${ }^{2}$ Department of Urology, Erciyes University, Kayseri, Turkey
}

*Address for Correspondence: Numan Baydilli, MD, Erciyes University, Faculty of Medicine, Department of Urology, Köşk Mahallesi, Prof. Dr. Turhan Feyzioğlu Cad. No:42, 38039 Melikgazi, Kayseri, Turkey, Tel: +903522076666-21134;

Email: dr_numan38@hotmail.com

Submitted: 16 January 2018

Approved: 29 January 2018

Published: 30 January 2018

Copyright: @ 2018 Selvi l, et al. This is an open access article distributed under the Creative Commons Attribution License, which permits unrestricted use, distribution, and reproduction in any medium, provided the original work is properly cited

Keywords: Congenital megalourethra; Prune belly syndrome; Urogenital anomalies

Check for updates

\section{Abstract}

Introduction: Prune Belly syndrome is a disease characterized by abdominal muscle defect, bilateral cryptorchidsim and urinary system anomalies (reflux megaureter, hydronephrosis, etc.). Pulmonary, cardiac, and gastrointestinal anomalies may also be present. Management of these rare cases is very important. In this case, the clinical course of a patient with Prune Belly syndrome with megaurethra is presented.

Case: The patient from the first gestation and parturition with birth weight of $2500 \mathrm{~g}$ and 38 weeks was hospitalized because of the bilateral hydronephrosis. His physical examination revealed undescended testicles and a large penis. The abdominal muscles were not very atrophic. The size of the kidney was small, bilateral hydroureteronephrosis and wide posterior urethra on the ultrasound. Renal function tests were progressively disturbed and the patient underwent cystourethroscopy for diagnostic purposes in terms of posterior urethral valve. A large diverticulum was found in anterior urethra. Prune Belly Syndrome was thought because the orifices were in appearance of reflux. The vesicostomy was applied. After vesicostomy the renal function tests got better but he was hospitalized due to urosepsis two times. In cystoscopic examination, the diverticulum in the urethra was filled with urine and the drainage was very slow. Phimosis was opened with dorsal slit technique. Cutaneous urethrostomy was proximal to the anterior diverticulum.

Conclusion: Prune Belly syndrome should be considered in patients with megaurethra and postrenal or renal insufficiency although there are no obvious clinical findings. In Prune Belly cases, via a large penis with obstruction signs, anterior urethral diverticulum should be considered.

\section{Introduction}

Prune Belly syndrome (PBS), first described in 1839 by Frolich, is a very rare congenital abnormality [1]. It is seen in approximately 1 in 40.000 males [2]. Nowadays as a result of widespread use of antenatal ultrasound and termination of pregnancy in that cases, we rarely see this syndrome. The other known names of this syndrome are Eagle Barret Syndrome, Osler-Parker Syndrome, and Mesenchymal dysplasia syndrome. The classic form of this syndrome contains congenital absence of abdominal wall muscles with 'Prune-like' appearance, cryptorchidism and malformations of urogenital tract (reflux megaureter, hydronephrosis etc.). Moreover cardiac, skeletal (such as club foot, hip dislocation), and gastrointestinal anomalies (such as intestinal malrotation, imperforate anus) accompany this syndrome. The most feared feature is development of pulmonary hypoplasia because of its being mortal. We presented an uncommon case of PBS with megalourethra which is characterized by diffuse dilatation of the penile urethra.

\section{Case Report}

A term male neonate with birth weight of $2500 \mathrm{~g}$, born via normal vaginal delivery after 38 weeks of gestation was hospitalized because of bilateral hydronephrosis 
and renal failure condition. His medical and family histories were unremarkable and there was no known antenatal hydronephrosis background. His physical examination revealed bilateral undescended testicles and a large penis (Figures 1A-1C). The abdominal muscles were not very atrophic, but abdomen was flabby. Ultrasound examination revealed small kidneys, bilateral hydroureteronephrosis and wide posterior urethra. Renal function tests were progressively disturbed. Because voiding cystourethrogram could not be performed due to unsuccessful cateterization, the patient underwent cystourethroscopy for diagnostic purposes in terms of posterior urethral valve (PUV). In cystourethroscopy, a large diverticulum was found in the anterior urethra and ureteral orifices were in appearance of reflux.

PBS was thought due to flabby abdominal appearance, bilateral undescended testicles, bilateral hydronephrosis and megaureter. Vesicostomy was applied for draining the renal units (Figure 1D). After vesicostomy, renal function improved but he was hospitalized due to urosepsis two times in the follow-up period. In cystoscopic examination, performed after urosepsis healed, it was seen that the diverticulum in the anterior urethra was filled with urine and the drainage was very slow. Cutaneous urethrostomy was performed at the proximal of anterior urethral diverticulum to obtain a successful drainage (Figure 1E). Furthermore phimosis was opened with dorsal slit technique to lessen the frequency of urinary infection.

\section{Discussion}

While PBS in males has classic triad of partial or complete lack of abdominal muscle, cryptorchidism and urinary tract dilatation abnormalities, incomplete type, called Pseudo-prune belly syndrome, can be seen less in females in the rates of \% 3-4 [3]. The urologic manifestations of this syndrome were firstly described by Parker in 1895 [4]. It remains controversial whether PBS is a genetic disorder or a primary mesodermal defect. Investigated some single genes may be associated with these complex malformations, affecting different specific organs and body structures.

One of the main pathogenesis to explain this syndrome is that obstructed or dilated bladder obstacle normal testicular descensus. This causes reflux of urine into the ureters, resulting in bilateral hydronephrosis or megaureter and it eventually goes to renal insufficiency. The distended bladder may also cause pressure necrosis on the developing abdominal musculature. The expected results of this obstructive uropathy are oligohydramnios and pulmonary hypoplasia, which can facilitate the other related problems such as cardiac, skeletal and gastrointestinal anomalies.

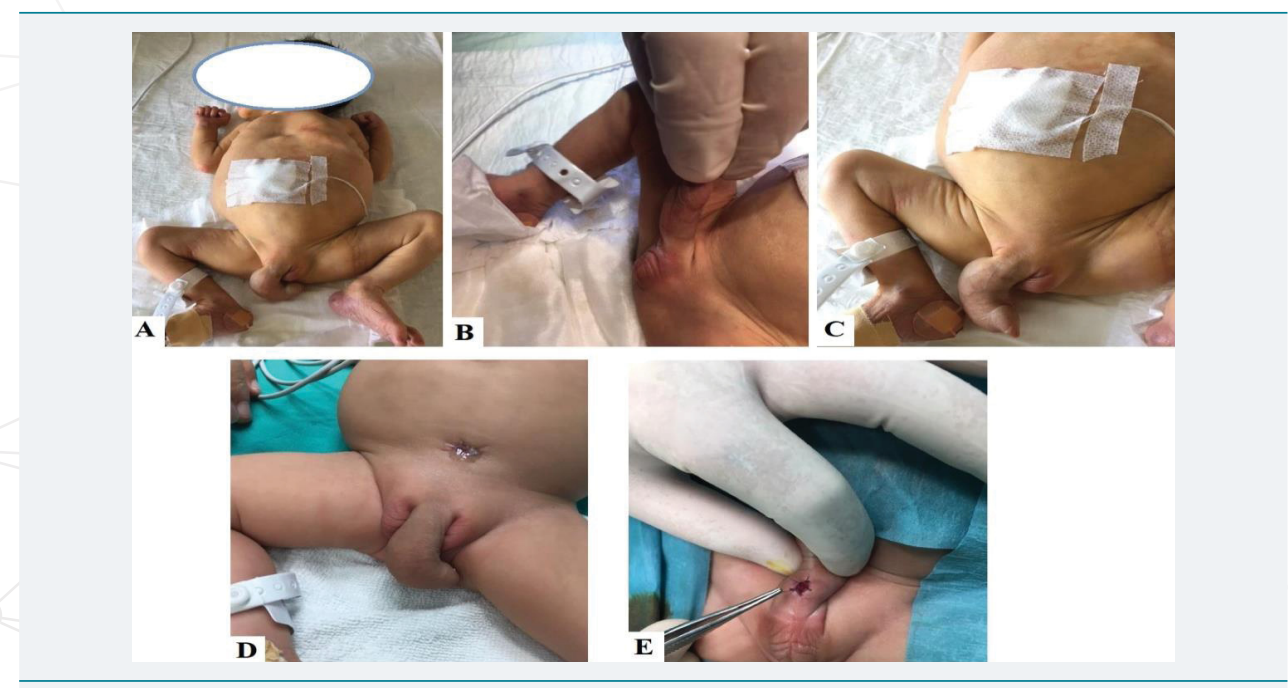

Figure 1: A: Patient's physical appearance, large penis takes attention. B: Appearance of undescended testicles. C: Megalouretra. D: Opened vesicostomy and megalourethra. E: Opened urethrostomy at the proximal of anterior urethral diverticulum. 
Diagnosis can be made by both prenatal and postnatal ultrasound. In prenatal ultrasound,presence ofdilatedand thin-walledbladder,bilateralhydroureteronephrosis and oligohydramnios are diagnostic for lower urinary tract obstruction. In the cases with megalourethra, the appearance of distended fluid-filled penis may be helpful in diagnosis. If the same findings such as bilateral hydroureteronephrosis is obtained in postnatal ultrasound, voiding cystourethrogram have to be performed to make a distinguish PUV. It also helps us to determine the presence of vesicoureteral reflux, urachal diverticulum and other urethral malformations such as megalourethra, urethral atresia. The main difference between PBS and PUV is presence of normally descended testes and thickened trabeculated bladder in the cases of PUV [5].

Congenital megalourethra results in diffuse dilatation of the anterior urethra and the pathogenesis is not secondary to distal obstruction. Generally, the etiology is associated with failure in the embryological development of corpus spongiosum. Traditionally, megalourethra is classified in two groups, including scaphoid and fusiform types [6]. Scaphoid type results in ventral sacculation of penile urethra and it is associated with absence of the corpus spongiosum. Conversely, absence or incomplete development of the erectile bodies can be seen in fusiform type. As a result, urethra may balloon dorsally as well [7].

As seen in our case, megalourethra in relation with PBS, is a rare seen finding and indicates a defect in development of mesoderm [8]. A mesodermal arrest, developed in the 3rd week of gestation, is blamed and this embryologic aberration elucidates all three parts of the triad in PBS.

The large sac like penile urethra can be ameliorated to a normal urethral appearance by surgical correction based on Nesbitt's technique [6]. As found in our patient, in the cases accompanied by urethral diverticulum, cutaneous urethrostomy can be temporarily performed to obtain a successful drainage.

\section{Conclusion}

PBS is a rare disease that can have terrible and mortal outcomes. Neonates with megalourethra and progressively postrenal failure should be considered in terms of PBS. The appearance of a large penis due to megalourethra may be a part of PBS. As our case, anterior urethral diverticulum accompanied with an obstruction sign causes a similar negative effect as PUV does and it deteriorates upper urinary system.

\section{References}

1. Kamra S, Santpur U, Puri N, Neetu Pippet. Prune belly syndrome: a case of early prenatal diagnosis. Int J Reprod Contracept Obstet Gynecol. 2017; 6: 1652-1655. Ref.: https://goo.gl/bDPLCu

2. Berrocal T, Loepez-Pereira P, Arjonilla A, Gutiérrez J. Anomalies of the distal ureter, bladder, and urethra in children: embryologic, radiologic, and pathologic features. Radiographics. 2002; 22: 11391164. Ref.: https://goo.gl/zBQkmQ

3. Rabinowitz R, Schillinger JF. Prune belly syndrome in the female subject. J Urol. 1977; 118: 454-456. Ref.: https://goo.gl/yA1ePi

4. Sellers BB Jr, McNeal R, Smith RV, Griswold WR, Mendoza SA. Congenital megalourethra associated with prune belly syndrome. J Urol. 1976; 116: 814-815. Ref.: https://goo.gl/6a3pM7

5. Rogers S, Sohaey R. Prune belly with megalourethra. Ultrasound Q. 2013; 29: 235-236. Ref.: https://goo.gl/Nn3mpd

6. Seki N, Senoh K, Kubo S, Tsunoda R. Congenital megalourethra: a case report. Int J Urol. 1998; 5: 191-193. Ref.: https://goo.gl/64ZUtv

7. Perrotin F, Ayeva-Derman M, Lardy H, Cloarec S, Lansac J, et al. Prenatal diagnosis and postnatal outcome of congenital megalourethra. Report of two cases. Fetal Diagn Ther. 2001; 16: 123-128. Ref.: https://goo.gl/JbtsWz

8. Bangroo AK, Tiwari S, Khetri R, Sahni M. Congenital pouch colon with prune belly syndrome and megalourethra. Pediatr Surg Int. 2005; 21: 474-477. Ref.: https://goo.gl/nau6PC 